\title{
Investigation of the structural characteristics of the electrodes of energy storage devices used in power plants based on renewable energy sources
}

\author{
Elena Kiseleva* and Fedor Lelin \\ Joint Institute for High Temperatures of the Russian Academy of Sciences, 125412, Izhorskaya st. 13 Bd.2, Moscow, Russia
}

\begin{abstract}
The porous structure of electrode materials was studied and an experimental analysis of the role of macropores in the electrodes of model double layer supercapacitors was carried out. It is shown that the excess value of the volume of macropores in the electrodes plays a negative role and, on the whole, in a complex manner, decreases the specific electrochemical characteristics of these devices. As a result of the research, high-efficiency activated carbons from wood waste, designed for use in supercapacitors, have been created. Correlations have been established between the parameters of the regime of thermochemical activation of wood, the porous structure of synthesized activated carbons and electrodes based on them and the specific characteristics of supercapacitors.
\end{abstract}

\section{Introduction}

At present, the problem of energy and resource saving both in Russia and in the world is quite acute. The growing demand for electrical energy makes it necessary to seek new sources of energy and optimize the energy consumption received from traditional systems. It is known that most renewable energy sources have significant daily and seasonal generation non-uniformities, and wind power installations are characterized by fluctuations with lifetimes from fractions of a few seconds [1]. If long peaks and recessions of power generation can be compensated by electrochemical accumulators, the double layered electrochemical supercapacitor (EDLC) is the optimal solution to improve the quality of the power supplied. The problem of suppressing fluctuations is also characteristic for diesel-generator sets, which form the basis of modern distributed generation

In some cases, an increase in the service life and cost reduction can be achieved by combining the batteries with EDLC in the power plant. Similar schemes are also used in electric vehicles [2].

In this scheme, the batteries provide power to the consumer in the basic mode in the absence of RES, and the transient processes are compensated by the EDLC [3]. Supercapacitors can also be used in power systems for those consumers whose electrical load is clearly impulsive [4]

The supercapacitor is a pulsed electrochemical device, which is primarily a power source. It differs from batteries of various types by a significantly lower energy intensity (units $\mathrm{W} \cdot \mathrm{h} / \mathrm{kg}$ ) and increased specific power $(2-10 \mathrm{~kW} / \mathrm{kg})$. The absence of Faraday processes in the operation of EDLC leads to an increase in its lifetime in comparison with accumulators, and also to the absence of restrictions on the depth of its discharge [5]. EDLCs are used to smooth out impulse loads, regenerate braking energy, or other similar processes that require high values of charge or discharge currents. For the satisfactory functioning of EDLC, it is necessary to have micro and mesopores in its active layers, which together result in a large value of the specific surface and, correspondingly, of the electric capacity. But with a charge (discharge) of high currents for a limited time, a situation may occur where a large fraction of the surface of the micropores turns out to be uncharged (undischarged).

What is the role of the macroporous electrode structure in solving this problem? It is generally believed that macropores create a channel in the active layer for the supply of electrolyte ions. But mesopores, and even micropores, also contribute to the value of the effective conductivity of the electrolyte. If we remove the macropores, then, as the estimates show, conductivity will decrease, but not catastrophically. In general, all three types of pores are usually present in EDLC electrodes: macropores, mesopores and micropores. In [6], structures with three types of pores were studied by the methods of the theory of porous electrodes and computer simulation. It follows from the theoretical model proposed in these studies that with a decrease in the proportion of macropores in the structure of the electrodes, the specific capacitance can increase substantially, even if the specific surface does not increase, and the effective conductivity of the electrolyte decreases. This result was analyzed in the present work on the basis of experimental data. 
Electrodes mainly determine the properties of supercapacitors. Therefore, the choice of electrode material and the technology of its preparation is extremely important for achieving high values of specific power and energy [7-8]. When using activated carbons (AC) for double-layer super-capacitor electrodes, the task of optimizing the porous structure of coal is actual. By varying the conditions for chemical activation, one can control the volume and distribution of pores by size, the nature and the number of functional groups on the surface. Earlier, the authors investigated the influence of the time and temperature of activation, the mass ratio of the activating agent and the raw material on the parameters of the porous structure, activated carbon: specific surface area, pore volume and radial distribution, and electrochemical characteristics of EDLC with aqueous (4.9 M sulfuric acid) and organic electrolytes (1M tetraethylammonium tetrafluoroborate) on the basis of these materials [9].

\section{Technique of the experiment}

From what has been said above, an important, as yet undefined problem, describing the effect on the value of the specific characteristics of EDLC of the content of macropores in the porous structure of $\mathrm{AC}$ is common to describe the principles of EDLC functioning. In order to demonstrate how the presence of a significant excessive amount of macropores in the AC affects the values of the specific characteristics of EDLC, the total pore volume was estimated in the work. In the electrode, it was evaluated using a bulk-weight method, and also from the mass and geometric volume of the dry electrode. In addition, the electrolyte volume in the electrodes was measured after measuring the characteristics of the model cells of the supercapacitors. Accordingly, the volume of macropores in the electrode was determined from the difference in the total measured volume, and the volume of micro- and mesopores determined from the LI method data.

When determining the volume of macropores in coal samples, it was found that the total volume of the measured pores of radius less than $100 \mathrm{~nm}$ by the method of limited evaporation is in close agreement with the data obtained by the volumetric-weight method. In addition, microphotographs of the corresponding increase in the particles synthesized in coal work do not observe macropores, unlike, for example, commercial coals from coconut shell.

In Figures 1 and 2, images of samples of coals Kuraray YP-50F and YP-80F obtained from the coconut shell are shown in addition to the images of one of the samples of the left-hand AC (Fig. 1 on the left and 2 on the left).

On this basis, we believe that in our activated coals the share of macropores is negligible. At the same time, in electrodes, the macropores formed by the interstices between the coal particles (and, to a lesser extent, the binder) are practically unavoidable. The total amount of electrolyte in the electrode based on $\mathrm{AC}$ from alder, concentrated in micro-, meso- and macropores, according to our estimates, should be $1.4 \mathrm{~cm} 3 / \mathrm{g}$ for maximum performance. In this case, the meso- and macropores of the electrode, which are the interstices between the particles, ensure the transport accessibility of the ions to each particle. Excessive amount of macropores and, accordingly, electrolyte in them are ballast. These pores do not contribute to the formation of DES, and the mass and volume of the electrolyte worsen the specific mass and volume characteristics of supercapacitors.

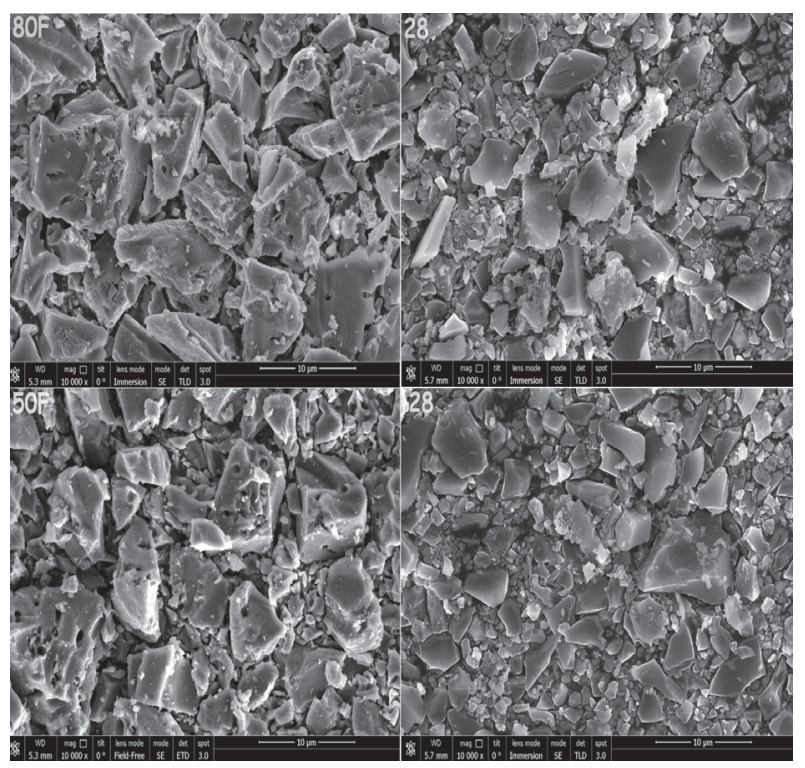

Fig. 1. Microphotographs of Kuraray YP-50F, YP-80F coal particles on the left, AC from waste wood on the right.

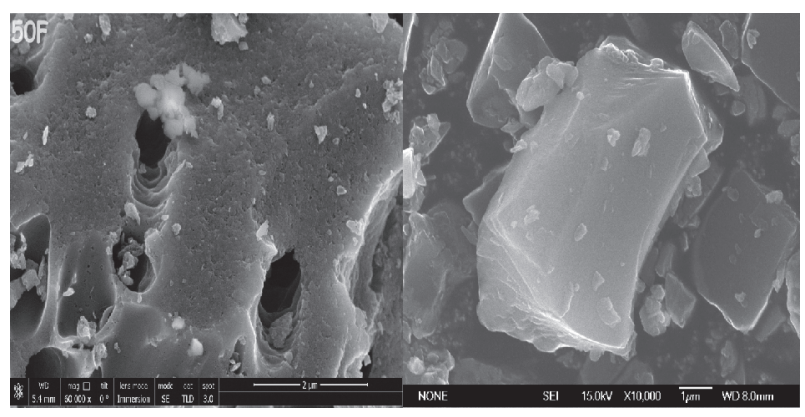

Fig. 2. Microphotographs of the Kuraray YP-50F coal particle on the left, AC from the wood waste on the right.

The conclusions drawn for the electrodes can be confirmed experimentally. Below (Figure 3) presents the experimental results for a large series of different samples of electrodes and activated coals due to the effect of the presence of macropores on the electrochemical characteristics of DSC. As mentioned above, the electrodes were made by calendering. This method of making electrodes does not completely eliminate macropores. These results represent a statistical treatment of a large number of independent experiments conducted over a long period of time, mainly to study the effect of synthesis parameters on the electrochemical characteristics of coals. In addition, the features of the electrode manufacturing technology could vary, the size of the coal particles, the binder content, the soot additives, etc., could vary. Thus, the volume of 
macropores in each electrode was a consequence of heterogeneous and often random causes.

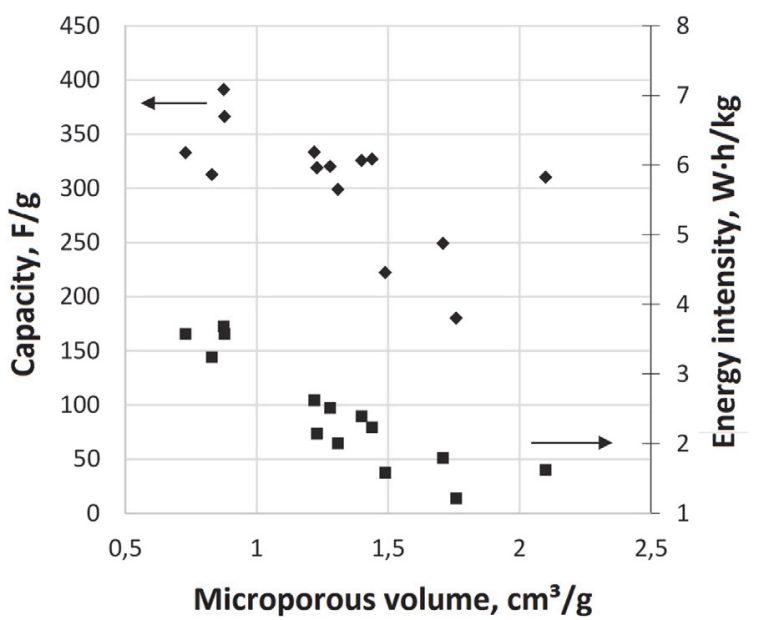

Fig. 3. Dependence of the specific energy intensity in relation to the total mass of electrodes and electrolyte and - the electrical capacitance from the volume of macropores in the DSC electrode.

In Figure 3 shows the experimental correlation dependences: along the $\mathrm{X}$ axis, the values of the volume of macropores in the electrode are plotted, and along the $\mathrm{Y}$ axis the specific energy intensity (right axis), calculated on the total mass of electrodes with electrolyte and specific electric capacity (left axis) per gram of dry activated carbon . Despite the scatter in the light of the foregoing, the graphs clearly show that an increase in the volume of macropores is accompanied by a decrease in specific electrochemical characteristics.

A partial decrease in the energy intensity in Figure 3 is easy enough to explain. Since this value was calculated based on the total mass of the electrodes with the electrolyte, the more the macropores in the electrode, the greater the amount of electrolyte it contains, the higher the denominator, and the lower the specific characteristics. After all, the contribution to the surface and, accordingly, to the capacity of the DPP, macropores practically do not contribute. Nevertheless, all the decrease in the specific energy intensity (up to three times) only due to the increase in the denominator of the electrolyte mass (about 2.5 times), taking into account the mass of the electrode, can not be explained.

As for the electric capacity per dry weight of coal, here, based on general considerations, the connection with the macropores would have to be more inverse, since macropores increase the effective conductivity of the electrolyte. Apparently, such a porous system is realized in which the excess volume of macropores prevents the charge and discharge of a part of the coal particles, contributing to their isolation from other particles. In view of the fact that in the system besides macropores there are filaments and aggregates of fluoroplastic particles, more isolated particles may have poor electrical contact over the solid phase of the carbon material. If, during a charge, these difficulties can be at least partially compensated by the exposure at the maximum voltage (which is realized in practice when the supercapacitors in the working circuit are under voltage), then it is impossible to influence the discharge for this purpose.

Thus, as a working hypothesis, it can be assumed that, with an excess of macropores, some of the micropores in the more isolated particles remain uncharged or under-discharged. As a consequence, there is a noticeable drop in capacity per dry weight of coal with an increase in the proportion of macropores. In this case, the more correct characteristic, on which the capacity depends, should be not the volume, but the surface of macropores and fluoroplastic aggregates.

\section{Conclusion}

As a result of the research, high-efficiency activated carbons from wood waste, designed for use in supercapacitors, have been created. Correlations between the parameters of the regime of thermochemical activation of wood, the porous structure of synthesized activated carbons and electrodes based on them and the specific characteristics of supercapacitors are established.

The main part of the work is devoted to the determination of the optimal porous structure of the activated activated coals. It is shown that the main pore volume falls to a radius of about $1 \mathrm{~nm}$. At the same time, the volume of the pores of these sizes at the level of 0.6 $\mathrm{cm} 3 / \mathrm{g}$ is sufficient to ensure the high energy characteristics of supercapacitors. A high specific capacitance of a double electric layer at a level of $390 \mathrm{~F} / \mathrm{g}$ in supercapacitors with sulfuric acid as an electrolyte was obtained. This value is a very high indicator, corresponding and even surpassing the world level.

In our opinion, obtaining such high specific characteristics became possible mainly due to the possibility of operative control of the parameters of the porous structure of the synthesized carbon materials achieved by the method of Limited Evaporation. At the same time, two main goals were pursued when choosing the main parameters of synthesis of AC: first, it was necessary to achieve the highest possible saturation of the specific volume of AC with micropores and mesopores, and secondly, it was required to reduce in every possible way the amount of excess ballast electrolyte in the AC.

This research has been supported by Russian Scientific Foundation (project No.11-50-00124) in part of activated carbon synthesis, electrode preparation routine, scanning electron microscopy.

\section{References}

1. W.F. Pickard, A.Q. Shen, N.J. Hansing, J. Renew. Sustain. Energy, 13, 1934-1945 (2009)

2. A.W. Stienecker, T. Stuart, C. Ashtiani, J. Power Sources, 154 (2), 561-566 (2006)

3. A. Andreotti, F. Mottola, M. Pagano, G. Velotto, Electric Power Systems Research, 78 (6), 10381046 (2008) 
4. A. Hande, T. Polk, W. Walker, D. Bhatia, Microprocessors and Microsystems, 31, 420-432 (2007)

5. K.K. Dyshchikov, Optimization of the interaction of nanostructured carbon materials and electrolytes based on ionic liquids to improve the electrical characteristics of supercapacitors, (RUSNANOFORUM, Moscow, 2008)

6. Yu.G. Chirkov, V.I. Rostokin, J. Electrochem, 50, 208-222 (2014)

7. M.A Zhurilova, I.A. Yanilkin, E.A. Kiseleva, I.N. Atamanyuk, E.I. Shkolnikov, Izv. Vyssh. Uchebn. Zaved., Khim. Khim. Tekhnol, 60 (4), 82-87 (2017)

8. E.A. Kiseleva, F.V. Lelin, M.A. Zhurilova, E.I. Shkolnikov, RUSS J APPL CHEM, 90 (5), 712715 (2017)

9. E.I. Shkolnikov, E.A. Kiseleva, D.E. Vervikishko, S.A. Kochanova, and E.V. Sidorova, RUSS J APPL CHEM, 90 (4), 547-552 (2017) 